\title{
乌ु
}

\section{Optical Polarization of Nuclear Spins in Silicon Carbide}

\author{
Abram L. Falk, ${ }^{1,2}$ Paul V. Klimov, ${ }^{1,3}$ Viktor Ivády, ${ }^{4,5}$ Krisztián Szász ${ }^{4,6}$ David J. Christle, ${ }^{1,3}$ \\ William F. Koehl, ${ }^{1}$ Ádám Gali, ${ }^{4,7}$ and David D. Awschalom ${ }^{1, *}$ \\ ${ }^{1}$ Institute for Molecular Engineering, University of Chicago, Chicago, Illinois 60637, USA \\ ${ }^{2}$ IBM T. J. Watson Research Center, Yorktown Heights, New York 10598, USA \\ ${ }^{3}$ Department of Physics, University of California, Santa Barbara, Santa Barbara, California 93106, USA \\ ${ }^{4}$ Institute for Solid State Physics and Optics, Wigner Research Centre for Physics, \\ Hungarian Academy of Sciences, H-1525 Budapest, Hungary \\ ${ }^{5}$ Department of Physics, Chemistry, and Biology, Linköping University, SE-581 83 Linköping, Sweden \\ ${ }^{6}$ Institute of Physics, Loránd Eötvös University, H-1117 Budapest, Hungary \\ ${ }^{7}$ Department of Atomic Physics, Budapest University of Technology and Economics, H-1111 Budapest, Hungary \\ (Received 22 December 2014; revised manuscript received 21 March 2015; published 17 June 2015)
}

We demonstrate optically pumped dynamic nuclear polarization of ${ }^{29} \mathrm{Si}$ nuclear spins that are strongly coupled to paramagnetic color centers in $4 \mathrm{H}$ - and $6 \mathrm{H}$-SiC. The $99 \% \pm 1 \%$ degree of polarization that we observe at room temperature corresponds to an effective nuclear temperature of $5 \mu \mathrm{K}$. By combining $a b$ initio theory with the experimental identification of the color centers' optically excited states, we quantitatively model how the polarization derives from hyperfine-mediated level anticrossings. These results lay a foundation for $\mathrm{SiC}$-based quantum memories, nuclear gyroscopes, and hyperpolarized probes for magnetic resonance imaging.

DOI: 10.1103/PhysRevLett.114.247603

PACS numbers: 76.70.Fz, 61.72.jn, 71.55.-i, 76.30.Mi

Silicon carbide is a promising material for quantum electronics at the wafer scale. It is both an industrially important substrate for high-performance electronic devices [1] and a host to several types of vacancy-related paramagnetic color centers with remarkable attributes [2-23]. Much like the diamond nitrogen-vacancy center $[24,25]$, these color centers have electronic spin states that can be addressed at either ensemble or single-spin levels $[18,19]$ through optically detected magnetic resonance (ODMR). Moreover, spin coherence times can exceed $1 \mathrm{~ms}$ [18], and ODMR can persist up to room temperature $[10,11,14,19]$. Although the fluctuating nuclear spin bath is a principal source of electronic spin decoherence in these types of systems [26], nuclear spins in $\mathrm{SiC}$ are not purely detrimental. If polarized and controlled, they would be a technologically valuable resource.

In this Letter, we show that near-infrared light can nearly completely polarize populations of ${ }^{29} \mathrm{Si}$ nuclear spins in SiC. In this dynamic nuclear polarization (DNP) [27,28] process, the optically pumped polarization of electron spins bound to either neutral divacancy $[4,5,8,10,14]$ or PL6 $[10,14,16]$ color centers is transferred to proximate nuclei via the hyperfine interaction. Optically polarizing nuclei in $\mathrm{SiC}$ is experimentally straightforward, requiring only broadband illumination and a small external magnetic field (300-500 G), with which we tune color-center ensembles to their ground-state (GS) or excited-state (ES) spin-level anticrossings (the GSLAC and ESLAC, respectively). Optically pumping crystals has previously led to roomtemperature DNP in napthalene [29], diamond [30-35], and GaNAs [36]. Our results show that room-temperature
DNP can be efficiently driven in a material that plays a leading role in the semiconductor industry.

We find that $\mathrm{SiC}$ color centers can mediate a high degree $(>85 \%)$ of ESLAC-derived nuclear polarization from at least 5 to $298 \mathrm{~K}$, a surprisingly broad temperature range for this mechanism. This robust DNP could be applied to initialize quantum memories in quantumcommunication technologies, especially since the color centers are telecom-range emitters, with narrow optical linewidths at low temperatures [16,21,37]. Other applications of DNP, including solid-state nuclear gyroscopes $[38,39]$ and entanglement-enhanced metrological devices [40], can employ SiC's long nuclear spin-lattice relaxation times [27,41] and its amenability to sophisticated growth and device fabrication.

The divacancy defect in $\mathrm{SiC}$ is a silicon vacancy $\left(V_{\mathrm{Si}}\right)$ adjacent to a carbon vacancy $\left(V_{C}\right)$ [Fig. 1(a)]. Among its several inequivalent forms, those aligned to the crystal's $c$ axis have the same $C_{3 v}$ symmetry as the nitrogen-vacancy center in diamond. They are the $h h$ and $k k$ divacancies in $4 H-\mathrm{SiC}[4,5]$, and the $h h, k_{1} k_{1}$, and $k_{2} k_{2}$ divacancies in $6 H-\mathrm{SiC}[14,42,43]$, where the $h$ (hexagonal site) and $k$ (quasicubic site) labels represent the inequivalent lattice sites for vacancies in the $\mathrm{SiC}$ lattice. The physical structure of the $c$-axis-oriented PL6 defect in $4 \mathrm{H}$-SiC $[10,14,16]$ is currently undetermined, but a close relationship to the neutral divacancies is indicated by its similar optical and spin resonances [10], radiative lifetimes [16], and hyperfine spectrum (measured here, see Table I). In the GS, these defects are spin triplets $(S=1)$ with the Hamiltonian 

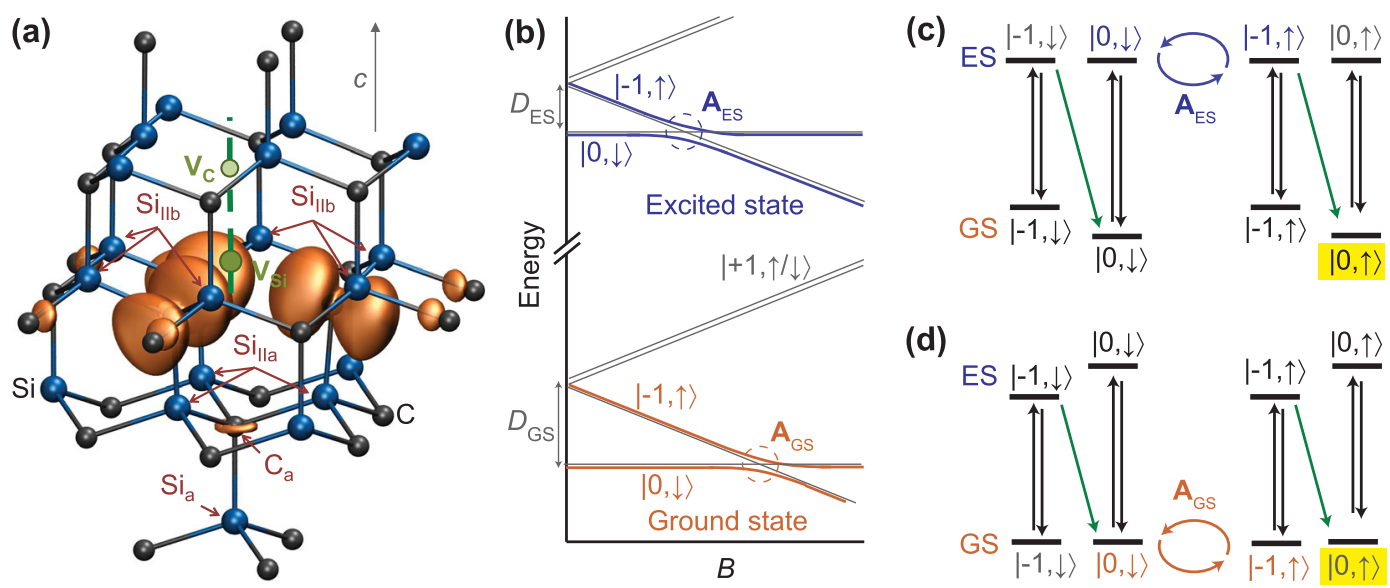

FIG. 1 (color). (a) An illustration of the $k_{1} k_{1}$ divacancy (green circles) in $6 H$-SiC. The calculated spin density is represented by orange-lobe isosurfaces and is primarily localized at the dangling bonds of the Si vacancy's nearest C atoms. We measure the DNP of ${ }^{29} \mathrm{Si}$ nuclei at the $\mathrm{Si}_{\text {III }}$ and $\mathrm{Si}_{\text {IIb }}$ sites. (b) Evolution of the ES and GS spin-sublevel energies with $B$, showing hyperfine-mediated hybridization of the electronic and nuclear spin sublevels at the ESLAC and GSLAC. The states drawn in gray do not hybridize. (c) For ESLAC-derived DNP, a hyperfine interaction in the ES causes $|0, \downarrow\rangle$ to partially evolve into $\mid-1$, $\uparrow\rangle$ every optical cycle. Together with the electron-spin polarization provided by the intersystem crossing (green arrows), this interaction causes optical cycling (black arrows) to polarize arbitrary $\left|m_{S}, m_{I}\right\rangle$ states into $|0, \uparrow\rangle$ (highlighted). The $m_{s}=+1$ electronic spin states do not participate in this process. (d) For GSLAC-derived DNP, the mechanism is the same as in (c), but the relevant hyperfine interaction is in the GS.

$$
\begin{aligned}
H_{\mathrm{GS}} & =\mu_{B} \mathbf{B} \cdot \mathbf{g}_{\mathrm{GS}} \cdot \mathbf{S}+D_{\mathrm{GS}} S_{\mathrm{Z}}^{2} \\
& +\sum_{j} \gamma_{j} \mathbf{B} \cdot \mathbf{I}_{j}+\mathbf{S} \cdot \mathbf{A}_{j, \mathrm{GS}} \cdot \mathbf{I}_{j},
\end{aligned}
$$

where $\mathbf{S}$ is the vector of spin-1 matrices, $\mathbf{g}_{\mathrm{GS}}$ is the electronic $g$ tensor, $\mu_{B}$ the Bohr magneton, $\mathbf{B}$ is the external magnetic field, $D_{\mathrm{GS}}$ is the electronic zero-field splitting parameter, and $\mathbf{A}_{j, \mathrm{GS}}$ the hyperfine tensor coupling the $j$ 'th nearby nucleus with spin $\mathbf{I}_{j}$ and gyromagnetic ratio $\gamma_{j}$. From left to right, the four terms in Eq. (1) represent the electronic Zeeman effect, the electronic crystal-field splitting, the nuclear Zeeman effect, and the hyperfine interaction between electronic and nuclear spins. At elevated temperatures, the form of the ES Hamiltonian is similar to that of the GS, with $g_{\mathrm{ES}}$ substituting for $g_{\mathrm{GS}}, D_{\mathrm{ES}}$ substituting for $D_{\mathrm{GS}}$, and $\mathbf{A}_{j, \mathrm{ES}}$ substituting for $\mathbf{A}_{j, \mathrm{GS}}$.

Silicon's dominant isotope is ${ }^{28} \mathrm{Si}$, with zero nuclear spin, but the spin- $1 / 2$ isotope ${ }^{29} \mathrm{Si}$ also has a fairly high natural abundance of $4.7 \%$. We denote the state of a hyperfinecoupled spin pair as $\left|m_{S}, m_{I}\right\rangle$, where $m_{S} \in\{-1,0,1\}$ is the electronic spin state and $m_{I} \in\{\uparrow, \downarrow\}$ is the ${ }^{29}$ Si nuclear spin state. Before any optical pumping, the spin pairs are in a statistical mixture of all six $\left|m_{S}, m_{I}\right\rangle$ states.

Optical illumination polarizes the color centers' electronic spins into the $m_{S}=0$ sublevel [44], a consequence of a spin-dependent intersystem crossing $[8,14,18,21]$. The degree of optically pumped electronic polarization for divacancies in $\mathrm{SiC}$ is at least $60 \%$ [14]. Without hyperfine

TABLE I. Parameters for the $c$-axis-oriented neutral divacancies, PL6 defects, and coupled ${ }^{29} \mathrm{Si}$ nuclei. ZPL stands for the zerophonon line. Both $D_{\mathrm{GS}}$ and $D_{\mathrm{ES}}$ are positive [44]. All experimental parameters are measured at $T=20 \mathrm{~K}$, except for $D_{\mathrm{ES}}$ of PL6, where the room-temperature value is provided. The $D_{\mathrm{GS}}$ and $A_{\mathrm{zz}}$ parameters are calculated at $T=0 \mathrm{~K}$, using the method in Ref. [16]. We match the divacancy forms in $6 H-\mathrm{SiC}$ with their corresponding spin transitions by comparing the experimentally determined and calculated $D_{\mathrm{GS}}$ parameters. The $\mathrm{Si}_{\mathrm{IIa}}$ sites all have threefold degeneracy per defect, and the $\mathrm{Si}_{\mathrm{IIb}}$ sites all have sixfold degeneracy per

\begin{tabular}{|c|c|c|c|c|c|c|c|c|c|}
\hline Defect & $\begin{array}{l}\text { ZPL } \\
(\mathrm{eV})\end{array}$ & $\begin{array}{l}\text { Sign of } \\
\Delta \mathrm{PL}\end{array}$ & $\begin{array}{c}D_{\mathrm{ES}} \\
(\mathrm{GHz})\end{array}$ & $\begin{array}{c}D_{\mathrm{GS}} \\
(\mathrm{GHz})\end{array}$ & $\begin{array}{c}A_{\mathrm{zz}}\left(\mathrm{Si}_{\mathrm{IIa}}\right) \\
(\mathrm{MHz})\end{array}$ & $\begin{array}{c}A_{\mathrm{zz}}\left(\mathrm{Si}_{\mathrm{IIb}}\right) \\
(\mathrm{MHz})\end{array}$ & $\begin{array}{c}D_{\mathrm{GS}} \\
(\mathrm{GHZ})\end{array}$ & $\begin{array}{c}A_{\mathrm{zz}}\left(\mathrm{Si}_{\mathrm{IIa}}\right) \\
(\mathrm{MHz})\end{array}$ & $\begin{array}{c}A_{\mathrm{zz}}\left(\mathrm{Si}_{\mathrm{IIb}}\right) \\
(\mathrm{MHz})\end{array}$ \\
\hline & & & & \multicolumn{3}{|c|}{$4 H-\mathrm{SiC}$ experiment } & \multicolumn{3}{|c|}{$4 H-\mathrm{SiC}$ calculation } \\
\hline$h h \mathrm{diV}$ & 1.095 & + & 0.84 & 1.336 & 12.3 & 9.2 & 1.358 & 11.6 & 9.3 \\
\hline$k k \operatorname{diV}$ & 1.096 & - & 0.78 & 1.305 & 13.2 & 10.0 & 1.320 & 12.4 & 10.2 \\
\hline \multirow[t]{2}{*}{ PL6 } & 1.194 & - & 0.94 & 1.365 & 12.5 & 9.6 & $\ldots$ & $\ldots$ & $\ldots$ \\
\hline & & & & \multicolumn{3}{|c|}{$6 H$-SiC experiment } & \multicolumn{3}{|c|}{$6 H-\mathrm{SiC}$ calculation } \\
\hline$h h \mathrm{diV}$ & 1.092 & + & 0.85 & 1.334 & 12.5 & 9.2 & 1.350 & 11.8 & 9.6 \\
\hline$k_{1} k_{1} \mathrm{diV}$ & 1.088 & - & 0.75 & 1.300 & 12.7 & 10.0 & 1.300 & 12.7 & 10.5 \\
\hline$k_{2} k_{2} \mathrm{diV}$ & 1.134 & - & 0.95 & 1.347 & 13.3 & 9.2 & 1.380 & 11.8 & 9.7 \\
\hline
\end{tabular}
defect. 
interactions, optical cycling does not polarize nuclear spins and results in equal populations of $|0, \downarrow\rangle$ and $|0, \uparrow\rangle$ states. However, when the defects' spin sublevels are tuned to the level anticrossing of their $m_{s}=0$ and $m_{s}=-1$ states [either the ESLAC or GSLAC, see Fig. 1(b)], the hyperfine interaction hybridizes the $|0, \downarrow\rangle$ and $|-1, \uparrow\rangle$ states. In each optical cycle, a spin pair in the $|0, \downarrow\rangle$ state will then have a chance of evolving into $|-1, \uparrow\rangle$, i.e., having its electron and nuclear polarizations be exchanged. Subsequent optical cycles then reorient the electronic spins, polarizing $|-1, \uparrow\rangle$ states into $|0, \uparrow\rangle$. Meanwhile, conservation of angular momentum prevents $|0, \uparrow\rangle$ from mixing with $|-1, \downarrow\rangle$. Together, these processes can efficiently polarize arbitrary $\left|m_{S}, m_{I}\right\rangle$ states into $|0, \uparrow\rangle$ [Figs. 1(c) and 1(d)].

Our $4 \mathrm{H}$-SiC wafer (purchased from Cree, Inc.) has vacancy complexes intentionally incorporated during crystal growth [10]. In our 6H-SiC wafer (purchased from II-VI, Inc.), we implant the wafer with ${ }^{12} \mathrm{C}$ ions, creating vacancies. Annealing the wafer then causes the vacancies to migrate and to pair into divacancies [14]. For continuous-wave ODMR measurements, we use a $975-\mathrm{nm}$ laser to nonresonantly excite the electronic transitions of ensembles of defects in either a $4 H$ or $6 H$-SiC sample and an InGaAs photoreceiver to collect the entire spectrum of near-infrared photoluminescence (PL) emitted by the defects. We then use a shortterminated antenna under the sample to apply a microwave field, whose frequency $(f)$ we sweep. When $f$ is resonant with an electronic spin transition, the electronic spin is rotated from its optically initialized $\left(m_{s}=0\right)$ state towards $m_{s}= \pm 1$, causing changes to the PL intensity ( $\Delta \mathrm{PL}$ ). Although the inequivalent defect forms in each of our two wafers are simultaneously optically excited, their nondegenerate spin-transition frequencies allow each form to be independently addressed $[10,14,44]$.

Using low microwave-power ODMR, we observe that each electronic spin transition has a hyperfine structure [Figs. 2(a)-2(b)] comprising symmetric side peaks around a central transition frequency $\left(f_{0}\right)$. In accordance with Eq. (1), these side peaks are at frequencies $f_{0} \pm A_{\mathrm{zz}} / 2$, where $A_{\mathrm{zz}}$ is the $c$-axis projection of the hyperfine interaction between the electron spin and a nearby nucleus. The two strongest hyperfine interactions between ${ }^{29} \mathrm{Si}$ nuclei and neutral divacancies in $4 \mathrm{H}$-SiC are known to be at $12-13 \mathrm{MHz}$ (the $\mathrm{Si}_{\text {II }}$ lattice site, with threefold degeneracy) and 9-10 MHz (the $\mathrm{Si}_{\text {IIb }}$ lattice site, with sixfold degeneracy), with $A_{\mathrm{zz}}$ positive and both hyperfine tensors nearly isotropic [5]. These sites correspond to the $\mathrm{Si}$ atoms nearest to the $\mathrm{C}$ atoms on which the neutral divacancy's electronic spin density is localized [16] [Fig. 1(a)].

We find that the hyperfine spectra for neutral divacancies of $6 H-\mathrm{SiC}$ and the PL6 defects in $4 H-\mathrm{SiC}$ are nearly identical to the previously known spectra for neutral divacancies in $4 \mathrm{H}$-SiC [5]. The lattice-site degeneracies for all these defects, which we infer from the relative amplitudes of the hyperfine-side peaks, are identical. We
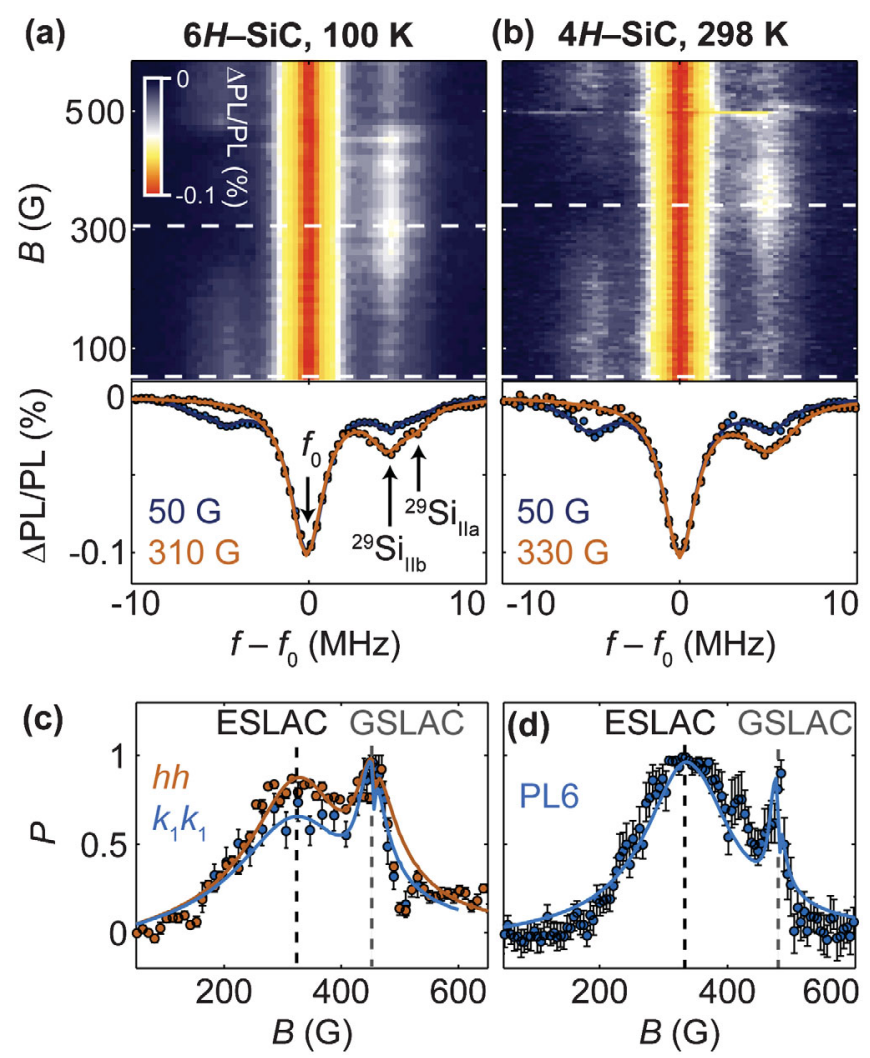

FIG. 2 (color). (a) Upper: Low-microwave-power ODMR spectrum of the $m_{s}=0$ to $m_{s}=1$ spin transition of the $h h$ divacancy in $6 H$-SiC at $T=100 \mathrm{~K}$. As $B$ varies from 50 to $650 \mathrm{G}, f_{0}$ varies from 1.5 to $3.2 \mathrm{GHz}$. Lower: Line cuts at the dashed white lines $(B=50$ and $B=310 \mathrm{G})$. The ${ }^{29} \mathrm{Si}$ nuclei at the $\mathrm{Si}_{\text {IIa }}$ and $\mathrm{Si}_{\text {IIb }}$ sites are unpolarized at $B=50 \mathrm{G}$ and nearly completely polarized at the ESLAC $(B=310 \mathrm{G})$. The continuous lines are fits to sums of Lorentzians [44]. (b) Upper: Lowpower ODMR spectrum of the $m_{s}=0$ to $m_{s}=1$ spin transition of the PL6 defects in $4 H-\mathrm{SiC}$ at $T=298 \mathrm{~K}$. Lower: Line cuts at $B=50$ and at $B=330 \mathrm{G}$, which is the PL6 ESLAC. (c) The nuclear polarization $(P)$ for ${ }^{29} \mathrm{Si}$ nuclei at the $\mathrm{Si}_{\mathrm{IIb}}$ sites of $h h$ and $k_{1} k_{1}$ divacancies in $6 H$-SiC at $T=100 \mathrm{~K}$, exhibiting peaks in $P$ at the ESLAC and GSLAC. $h h$ divacancies have stronger ESLAC-derived DNP than $k_{1} k_{1}$ divacancies. The error bars are single- $\sigma$ credible intervals set by the fits. The continuous lines are $P$ values simulated from our theoretical model, plotted with a resolution of $3 \mathrm{G}$. (d) $\mathrm{Si}_{\mathrm{IIb}}$ nuclear spin polarization for PL6coupled nuclei in $4 H-\mathrm{SiC}$ at $T=298 \mathrm{~K}$ (experiment and theory). The origin of the small peak in $P$ at $420 \mathrm{G}$ is unknown.

use electron spin echo envelope modulation [48] to refine our measurement of the hyperfine-interaction strengths [44]. Using $a b$ initio density-functional theory (DFT), we then calculate the hyperfine and $D_{\mathrm{GS}}$ constants for each form of $c$-axis-oriented neutral divacancy (Table I). These calculations implement the plane wave and projected augmented wave method [49-51], 576- and 432-atom supercells with $\Gamma$-point sampling of the Brillouin zone, and HSE06 and PBE functionals [16,52-55]. As has been previously done in $4 \mathrm{H}$-SiC [5], we compare theory and 
experiment to associate each divacancy form in $6 \mathrm{H}$-SiC with an experimentally observed spin resonance (Table I).

We define the degree of nuclear spin polarization $(P)$ as $P=\left(I^{+}-I^{-}\right) /\left(I^{+}+I^{-}\right)$, where $I^{+}$and $I^{-}$, respectively, represent the populations of ${ }^{29} \mathrm{Si}$-nuclear spins pointing $\uparrow$ and $\downarrow$ [32]. $P$ is defined separately for each pairing of inequivalent defect form with inequivalent ${ }^{29} \mathrm{Si}$ site. We quantify $P$ by performing a global fit the ODMR line shapes to the sum of seven Lorentzians, one centered at $f_{0}$ and one pair at each of the $\mathrm{Si}_{\text {IIa }}, \mathrm{Si}_{\mathrm{IIb}}$, and $\mathrm{C}_{\mathrm{II}}$ hyperfine resonances ([44]). For each resonance, we compute $P$ by inferring the relative amplitudes of each pair of Lorentzians (Fig. 2). Asymmetry in the intensities of the ODMR side peaks is thus the signature of nuclear polarization. We concentrate on DNP at the $\mathrm{Si}_{\text {IIb }}$ site, whose sixfold degeneracy results in the strongest ODMR signal.

Boltzmann statistics would require a sub-mK sample temperature $(T)$ for $P$ to exceed even a few percent. Indeed, at both low $(B<200 \mathrm{G})$ and high $(B>500 \mathrm{G})$ magnetic fields, we observe that $P$ is nearly zero. In the $200 \mathrm{G}<$ $B<500 \mathrm{G}$ regime, however, we observe strong DNP. For PL6 defects at room temperature and $B=330 \mathrm{G}, P$ reaches $99 \% \pm 1 \%$, an effective temperature of $5 \mu \mathrm{K}$ [Fig. 2(d)].

Two prominent peaks can be seen in $P$ as a function $B$, one centered at 300-335 and the other at 465-490 G [Figs. 2(c)-2(d)]. Anticipating that level anticrossings underlie the electron-to-nuclear polarization transfer, we hypothesize that these two peaks receptively correspond to the ESLAC and GSLAC. As expected, the higher $B$-value peaks in $P$ correspond precisely to $D_{\mathrm{GS}} /\left(g_{\mathrm{GS}} \mu_{B}\right)$ (Table I) for each defect form, indicating that they are associated with the GSLAC. Because of the short (14-ns) optical lifetimes of the metastable excited states [16], though, our low-microwave-power ODMR measurements rotate spins too slowly to show ES-spin transitions.

High-microwave-power ODMR reveals the spin-triplet electronic excited states [Figs. 3(a)-3(c)], with $g_{\mathrm{ES}}=2.0$ and $D_{\mathrm{ES}} /\left(g_{\mathrm{ES}} \mu_{B}\right)$ matching precisely with the lower- $B$ peaks in $P$ (Table I). Unlike the GS-ODMR transitions, which exhibit nonzero $\Delta \mathrm{PL}$ when microwaves and optical illumination are alternated (due to Rabi driving), they are only visible when microwaves and optical illumination are coincident [44], supporting their identification as ES resonances. Moreover, due to spin mixing in the GS, each divacancy's ES-ODMR signal has a minimum at its corresponding GSLAC [Fig. 3(d)], confirming the association between ES- and GS-spin transitions. Thus, peaks in $P$ [Figs. 2(c)-2(d)] correspond to GSLACs and ESLACs [Figs. 3(a)-3(c)].

To understand the DNP quantitatively, we simulate the optical polarization process using a recently developed model [56] of color-center-mediated DNP. This model simulates the nuclear polarization while taking into account the full hyperfine tensor and the simultaneous contributions from both ESLAC-and GSLAC-derived DNP
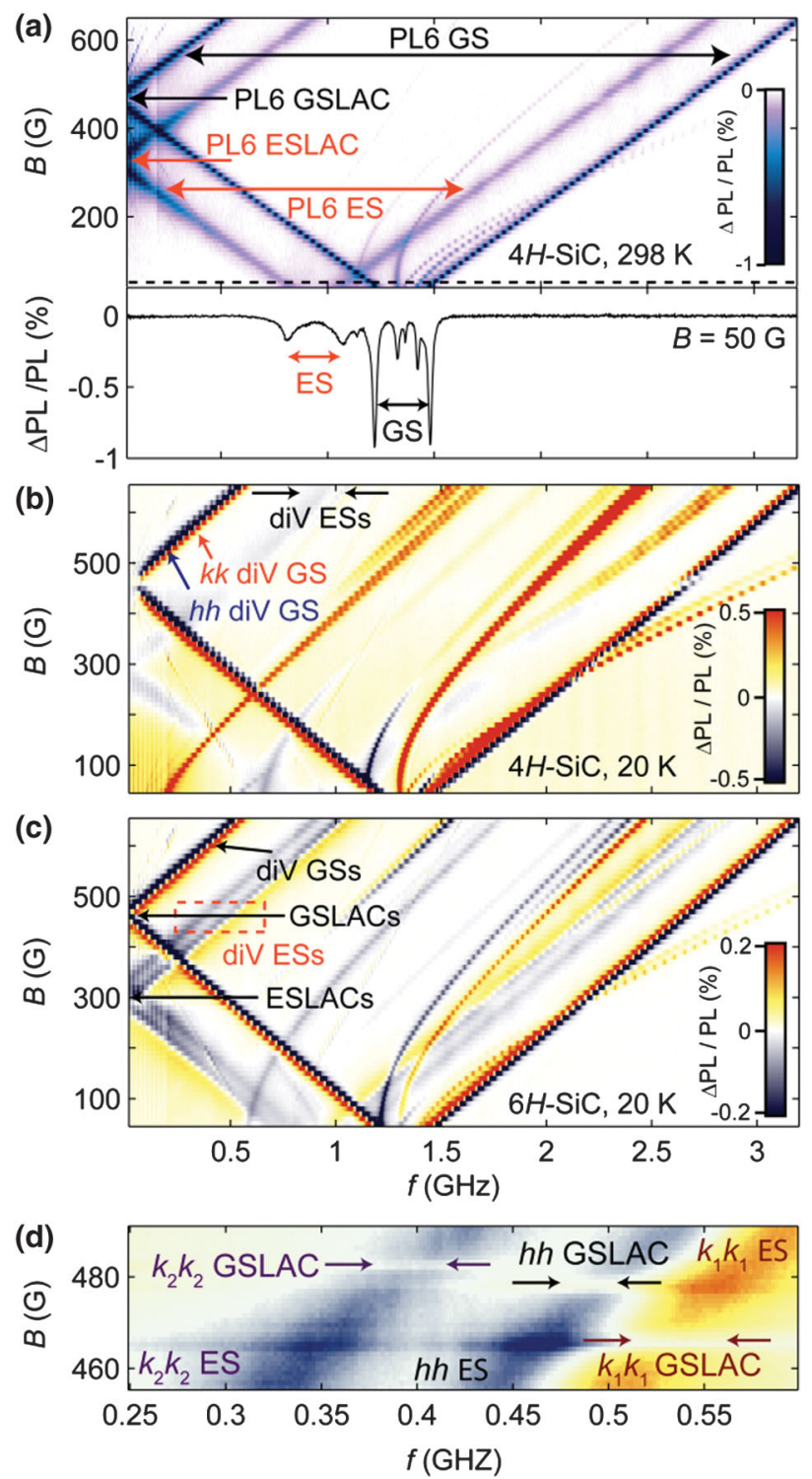

FIG. 3 (color). (a) Upper: Experimental high-power ODMR spectrum of PL6 at $T=298 \mathrm{~K}$. Lower: Line cut of the ODMR spectrum at $B=50 \mathrm{G}$ (the dashed line). The curved lines correspond to basal-plane oriented defects. The different divacancy forms have nondegenerate microwave transition frequencies and are therefore individually addressable. (b) High-power ODMR spectrum of the neutral divacancies in $4 \mathrm{H}$-SiC and (c) of the neutral divacancies in $6 H-\mathrm{SiC}$, all at $T=20 \mathrm{~K}$. (d) Zoom-in of the red dashed rectangle drawn in (c). Because of spin mixing in the GS, each divacancy's ES ODMR signal has a minimum at its corresponding GSLAC.

at intermediate $B$ values. In applying it, we use as many experimental parameters as possible, including electronic fine structure parameters, hyperfine-interaction strengths, and optical lifetimes (Table I and Ref. [16]). The orientation of the hyperfine tensors' principal axes are taken from our $a b$ initio simulations [16], and fitting parameters represent thermally driven depolarization of the nuclear spins and the effective electron-nuclear interaction times per optical 

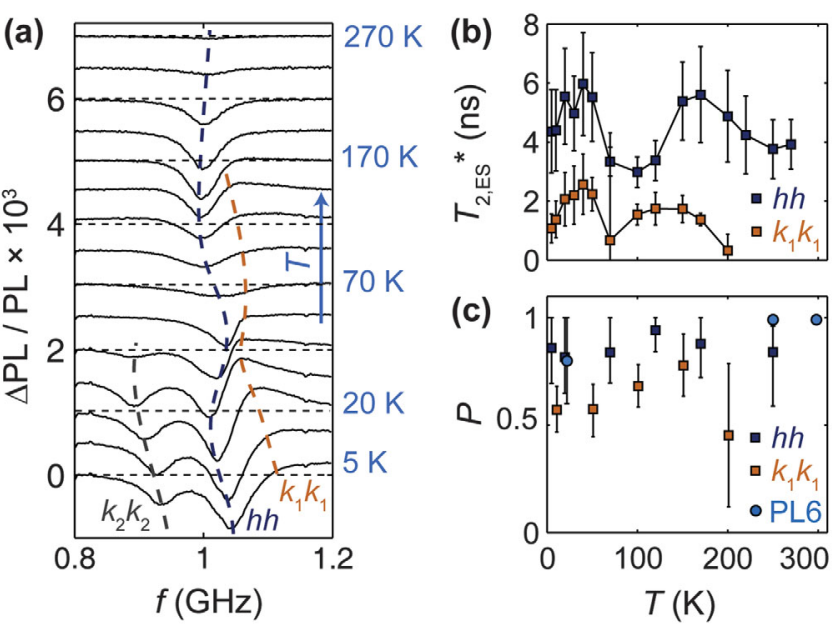

FIG. 4 (color). (a) Temperature dependence of the high-power ES ODMR for the three $c$-axis-oriented neutral divacancies in $6 H$-SiC at $B=650 \mathrm{G}$. The ES ODMR curves are sequentially offset from $\triangle \mathrm{PL}=0$, for clarity, and the bold lines are guides to the eye that follow the ES-spin resonances. (b) $T_{2, \mathrm{ES}}{ }^{*}$ for divacancies in $6 \mathrm{H}-\mathrm{SiC}$, calculated by fitting the curves in (a) to a sum of three Lorentzians and taking $T_{2, \mathrm{ES}}{ }^{*}$ to be $1 / \pi$ times the inverse of the full-width at half-maximum linewidths. The single$\sigma$ error bars derive from the fits. (c) Temperature dependence of $P$ at the $\mathrm{Si}_{\mathrm{IIb}}$ site of divacancies in $6 H$-SiC, and for PL6 in $4 H-\mathrm{SiC}$, when $B$ is tuned to the ESLAC (270-330 G). [44].

cycle. The modeled polarization and the experimental data show excellent agreement [Figs. 2(c)-2(d)].

Our model finds that effective electron-nuclear interaction times are primarily responsible for the differences in DNP efficiencies across the different defect types. Experimentally, we can use the ES spin-dephasing time $\left(T_{2, \mathrm{ES}}{ }^{*}\right)$ as a proxy for the electron-nuclear interaction time and estimate the $T_{2, \mathrm{ES}}{ }^{*}$ times as $1 / \pi$ times the inverse of the ES ODMR linewidths [Figs. 4(a)-4(b)]. As predicted, the $h h$ divacancy, whose ESLAC-derived nuclear polarization is stronger than that of the $k_{1} k_{1}$ divacancy [Fig. 2(c)], also has a longer $T_{2, \mathrm{ES}}{ }^{*}$ time. Moreover, comparing nonresonant ESLAC-derived DNP in SiC to that for nuclei coupled to diamond nitrogen-vacancy centers [30-35], we find that while both systems exhibit nearly ideal ESLAC-derived DNP at room temperature, the low-temperature DNP is significantly more robust in $\mathrm{SiC}$.

In diamond, both the nitrogen-vacancy center's ES-spin coherence and its off-resonantly pumped ESLAC-derived DNP rapidly decline below $T=50 \mathrm{~K}$ [34]. This diminishment is due to the deactivation of the dynamic Jahn-Teller effect, in which phonons motionally narrow pairs of ES electronic orbitals into a single coherent spin resonance [57-59]. In $\mathrm{SiC}$, at $T=5 \mathrm{~K}$, the base temperature of our cryostat, we observe both a coherent ES spin resonance [Figs. 4(a)-4(b)] and strong ESLAC-derived DNP ( $P=85 \% \pm 5 \%$ for the $h h$ divacancy). As $T$ is raised, however, strong DNP persists [Fig. 4(c)] while $D_{\mathrm{ES}}$ and
$T_{2, \text { ES }}{ }^{*}$ oscillate as a function of $T$. These behaviors suggest that while SiC Jahn-Teller effects play a role in the strong DNP, they are also complex and require further study through techniques like pulsed ODMR in the ES $[58,60]$.

Our results show that optical pumping can strongly polarize nuclear spins in $\mathrm{SiC}$. The identification of the ES-spin transitions provides insight into the DNP process and the electronic structure of $\mathrm{SiC}$ divacancies. We expect optically pumped DNP to generalize to nuclear spins at other sites, such as the $\mathrm{Si}_{\mathrm{a}}$ and $\mathrm{C}_{\mathrm{a}}$ sites, which lie on the divacancy's symmetry axis [see Fig. 1(a)], and to other color centers. Moreover, spin diffusion [35] may enhance the crystal's total nuclear spin polarization well above the $10^{16} \mathrm{~cm}^{-3}$ density [44] of strongly coupled nuclei that we can polarize in our samples. SiC nanostructures could then be used as hyperpolarized contrast agents in magnetic resonance imaging $[61,62] . \mathrm{SiC}$ is proving to have not only a key role in the power electronics and optoelectronics industries but also a promising future in the fields of spintronics, sensing, and quantum information.

The authors thank Viatcheslav V. Dobrovitski, Bob B. Buckley, F. Joseph Heremans, and Charles de las Casas for helpful conversations. This work is supported by the Air Force Office of Scientific Research (AFOSR), the AFOSR Multidisciplinary Research Program of the University Research Initiative, the National Science Foundation, the Material Research Science and Engineering Center, the Knut \& Alice Wallenberg Foundation "Isotopic Control for Ultimate Materials Properties," the Lendület program of the Hungarian Academy of Sciences, and the National Supercomputer Center in Sweden.

*awsch@uchicago.edu

[1] J. Rabkowski, D. Peftitsis, and H.-P. Nee, Industrial Electronics 6, 17 (2012).

[2] S. Castelletto and B. C. Johnson, Adv. Opt. Mater. 1, 609 (2013).

[3] W. E. Carlos, E. R. Glaser, and B. V. Shanabrook, Physica (Amsterdam) 340-342B, 151 (2003).

[4] P. G. Baranov, I. V. Il'in, E. N. Mokhov, M. V. Muzaforova, S. B. Orlinskii, and J. Schmidt, JETP Lett. 82, 441 (2005).

[5] N. T. Son, P. Carlsson, J. ul Hassan, E. Janzén, T. Umeda, J. Isoya, A. Gali, M. Bockstedte, N. Morishita, T. Ohshima, and H. Itoh, Phys. Rev. Lett. 96, 055501 (2006).

[6] J. v. Tol, G. W. Morley, S. Takahashi, D. R. McCamey, C. Boehme, and W. E. Zvanut, Appl. Magn. Reson. 36, 259 (2009).

[7] J. R. Weber, W. F. Koehl, J. B. Varley, A. Janotti, B. B. Buckley, C. G. V.d. Walle, and D. D. Awschalom, Proc. Natl. Acad. Sci. U.S.A. 107, 8513 (2010).

[8] A. Gali, Phys. Status Solidi B 248, 1337 (2011).

[9] P. G. Baranov, A. P. Bundakova, and A. A. Soltamov, S. B. Orlinskii, I. V. Borovykh, R. Zondervan, R. Verberk, and J. Schmidt, Phys. Rev. B 83, 125203 (2011). 
[10] W. F. Koehl, B. B. Buckley, F. J. Heremans, G. Calusine, and D. D. Awschalom, Nature (London) 479, 84 (2011).

[11] D.. Riedel, F. Fuchs, H. Kraus, S. Vath, A. Sperlich, V. Dyakonov, A. A. Soltamova, P. G. Baranov, V. A. Ilyin, and V. Astakhov, Phys. Rev. Lett. 109, 226402 (2012).

[12] V. A. Soltamov, A. A. Soltamova, P. G. Baranov, and I. I. Proskuryakov, Phys. Rev. Lett. 108, 226402 (2012).

[13] D. Riedel, F. Fuchs, H. Kraus, S. Väth, A. Sperlich, V. Dyakonov, A. A. Soltamova, P. G. Baranov, V. A. Ilyin, and G. V. Astakhov, Phys. Rev. Lett. 109, 226402 (2012).

[14] A. L. Falk, B. B. Buckley, G. Calusine, W. F. Koehl, V. V. Dobrovitski, A. Politi, C. A. Zorman, P. X.-L. Feng, and D. D. Awschalom, Nat. Commun. 4, 1819 (2013).

[15] S. Castelletto, B. C. Johnson, and A. Boretti, Adv. Opt. Mater. 1, 609 (2013).

[16] A. L. Falk, P. V. Klimov, B. B. Buckley, V. Ivády, I. A. Abrikosov, G. Calusine, W. F. Koehl, A. Gali, and D. D. Awschalom, Phys. Rev. Lett. 112, 187601 (2014).

[17] P. V. Klimov, A. L. Falk, B. B. Buckley, and D. D. Awschalom, Phys. Rev. Lett. 112, 087601 (2014).

[18] D. J. Christle, A. L. Falk, P. Andrich, P. V. Klimov, J. U. Hasan, N. T. Son, E. Janzen, T. Ohshima, and D. D. Awschalom, Nat. Mater. 14, 160 (2015).

[19] M. Widmann, S. Lee, T. Rendler, N. T. Son, H. Fedder, S. Paik, L. Yang, N. Zhao, S. Yang, I. Booker, A. Denisenko, M. Jamali, S. A. Momenzadeh, I. Gerhardt, T. Ohshima, A. Gali, E. Janzen, and J. Wrachtrup, Nat. Mater. 14, 164 (2015).

[20] H. Kraus, V. A. Soltamov, D. Riedel, S. Vath, F. Fuchs, A. Sperlich, P. G. Baranov, V. Dyakonov, and G. V. Astakhov, Nat. Phys. 10, 157 (2014).

[21] O. V. Zwier, D. O'Shea, A. R. Onur, and C. H. v. d. Wal, arXiv:1411.1366.

[22] A. Muzha, F. Fuchs, N. V. Tarakina, D. Simin, M. Trupke, V. A. Soltamov, N. N. Mokhov, P. G. Baranov, V. Dyakonov, A. Krueger, and G. V. Astakhov, Appl. Phys. Lett. 105, 243112 (2014).

[23] H. Kraus, V. A. Soltamov, F. Fuchs, D. Simin, A. Sperlich, P. G. Baranov, G. V. Astakhov, and V. Dyakonov, Sci. Rep. 4, 1 (2014).

[24] F. Jelezko, T. Gaebel, I. Popa, A. Gruber, and J. Wrachtrup, Phys. Rev. Lett. 92, 076401 (2004).

[25] V. V. Dobrovitski, G. D. Fuchs, A. L. Falk, C. Santori, and D. D. Awschalom, Annu. Rev. Cond. Mater. Phys. 4, 23 (2013).

[26] L.-P. Yang, C. Burk, M. Widmann, S.-Y. Lee, J. Wrachtrup, and N. Zhao, Phys. Rev. B 90, 241203 (2014).

[27] G. E. G. Hardeman, J. Phys. Chem. Solids 24, 1223 (1963).

[28] G. Lampel, Phys. Rev. Lett. 20, 491 (1968).

[29] A. Henstra, T.-S. Lin, J. Schmidt, and W. T. Wenckebach, Chem. Phys. Lett. 165, 6 (1990).

[30] X.-F. He, N. B. Manson, and P. T. H. Fisk, Phys. Rev. B 47, 8809 (1993).

[31] B. Smeltzer, J. McIntyre, and L. Childress, Phys. Rev. A 80, 050302 (2009).

[32] V. Jacques, P. Neumann, J. Beck, M. Markham, D. Twitchen, J. Meijer, F. Kaiser, G. Balasubramanian, F. Jelezko, and J. Wrachtrup, Phys. Rev. Lett. 102, 057403 (2009).

[33] B. Smeltzer, L. Childress, and A. Gali, New J. Phys. 13, 025021 (2011).

[34] R. Fischer, A. Jarmola, P. Kehayias, and D. Budker, Phys. Rev. B 87, 125207 (2013).
[35] R. Fischer, C. O. Bretschneider, P. London, D. Budker, D. Gershoni, and L. Frydman, Phys. Rev. Lett. 111, 057601 (2013).

[36] Y. Puttisong, X. J. Wang, I. A. Buyanova, J. Geelhaar, H. Riechert, A. J. Ptak, C. W. Tu, and W. M. Chen, Nat. Commun. 4, 1751 (2013).

[37] B. Magnusson and E. Janzen, Mater. Sci. Forum 483-485, 341 (2005).

[38] A. Ajoy and P. Cappellaro, Phys. Rev. A 86, 062104 (2012).

[39] M. P. Ledbetter, K. Jensen, R. Fischer, A. Jarmola, and D. Budker, Phys. Rev. A 86, 052116 (2012).

[40] V. Giovannetti, S. Lloyd, and L. Maccone, Nat. Photonics 5, 222 (2011).

[41] J. S. Hartman, A. Narayanan, and Y. Wang, J. Am. Chem. Soc. 116, 4019 (1994).

[42] N. T. Son, P. N. Hai, M. t. Wagner, W. M. Chen, A. Ellsion, C. Hallin, B. Monemar, and E. Janzén, Semicond. Sci. Technol. 14, 1141 (1999).

[43] L. Patrick and J. Choyke, Phys. Rev. B 5, 3253 (1972).

[44] See Supplemental Material at http://link.aps.org/ supplemental/10.1103/PhysRevLett.114.247603 for additional experimental and theoretical methods, including the calculation of the nuclear spin concentration, and our Bayesian approach to fitting the nuclear spin polarization at multiple atomic sites, which includes Refs. [45-47].

[45] E. V. Oort and M. Glasbeek, Chem. Phys. 143, 131 (1990).

[46] V. V. Dobrovitski, A. E. Feiguin, D. D. Awschalom, and R. Hanson, Phys. Rev. B 77, 245212 (2008).

[47] E. Laloy and J. A. Vrugt, Water Res. Res. 48, W01526 (2012).

[48] W. B. Mims, K. Nassau, and J. D. McGee, Phys. Rev. 123, 2059 (1961).

[49] G. Kresse and J. Hafner, Phys. Rev. B 49, 14251 (1994).

[50] G. Kresse and J. Furthmüller, Phys. Rev. B 54, 11169 (1996).

[51] P. E. Blochl, Phys. Rev. B 50, 17953 (1994).

[52] J. P. Perdew, K. Burke, and M. Ernzerhof, Phys. Rev. Lett. 77, 3865 (1996).

[53] J. Heyd and G. Scuseria, J. Chem. Phys. 121, 1187 (2004).

[54] A. F. Izmaylov, G. Scuseria, and M. J. Frisch, J. Chem. Phys. 125, 104103 (2006).

[55] K. Szász, T. Hornos, M. Marsman, and A. Gali, Phys. Rev. B 88, 075202 (2013).

[56] V. Ivády, K. Szász, A. L. Falk, P. V. Klimov, D. J. Christle, E. Janzén, I. A. Abrikosov, D. D. Awschalom, and A. Gali, arXiv:1505.05651.

[57] K.-M. C. Fu, C. Santori, P. E. Barclay, L. J. Rogers, N. B. Manson, and R. G. Beausoleil, Proc. SPIE Int. Soc. Opt. Eng. 7611, 761108 (2010).

[58] G. D. Fuchs, V. V. Dobrovitski, D. M. Toyli, F. J. Heremans, C. D. Weis, T. Schenkel, and D. D. Awschalom, Nat. Phys. 6, 668 (2010).

[59] G. D. Fuchs, V. V. Dobrovitski, R. Hanson, A. Batra, C. D. Weis, T. Schenkel, and D. D. Awschalom, Phys. Rev. Lett. 101, 117601 (2008).

[60] G. D. Fuchs, A. L. Falk, V. V. Dobrovitski, and D. D. Awschalom, Phys. Rev. Lett. 108, 157602 (2012).

[61] E. Terreno, D. D. Castelli, A. Viale, and S. Aime, Chem. Rev. 110, 3019 (2010).

[62] M. C. Cassidy, H. R. Chan, B. D. Ross, P. K. Bhattacharya, and C. M. Marcus, Nat. Nanotechnol. 8, 363 (2013). 